Kim A. Meijer, MSc

Anand J.C. Eijlers, MD

Linda Douw, $\mathrm{PhD}$

Bernard M.J. Uitdehaag, MD

Frederik Barkhof, MD

Jeroen J.G. Geurts, PhD

Menno M. Schoonheim,

$\mathrm{PhD}$

Correspondence to

Kim A. Meijer:

k.meijer@vumc.nl
Supplemental data at Neurology.org

\title{
Increased connectivity of hub networks and cognitive impairment in multiple sclerosis
}

\section{ABSTRACT}

Objective: To investigate default-mode network (DMN) and frontoparietal network (FPN) dysfunction in cognitively impaired (CI) patients with multiple sclerosis (MS) because these networks strongly relate to cognition and contain most of the hubs of the brain.

Methods: Resting-state fMRI and neuropsychological assessments were performed in 322 patients with MS and 96 healthy controls (HCs). Patients with MS were classified as $\mathrm{Cl}$ ( $z$ score $<-2.0$ on at least 2 tests; $n=87$ ), mildly cognitively impaired ( $z$ score $<-1.5$ on at least 2 tests and not $\mathrm{Cl} ; \mathrm{n}=65)$, and cognitively preserved ( $\mathrm{CP} ; \mathrm{n}=180$ ). Within-network connectivity, connectivity with the rest of the brain, and between-network connectivity were calculated and compared between groups. Connectivity values were normalized for individual means and SDs.

Results: Only in $\mathrm{Cl}$, both the DMN and FPN showed increased connectivity with the rest of the brain compared to $\mathrm{HCs}$ and $\mathrm{CP}$, with no change in within- or between-network connectivity. Regionally, this increased connectivity was driven by the inferior parietal, posterior cingulate, and angular gyri. Increased connectivity with the rest of the brain correlated with worse cognitive performance, namely attention for the FPN as well as information processing speed and working memory for both networks.

Conclusions: $\mathrm{In} \mathrm{Cl}$ patients with MS, the DMN and FPN showed increased connectivity with the rest of the brain, while normal within- and between-network connectivity levels were maintained. These findings indicate that cognitive impairment in MS features disturbed communication of hub-rich networks, but only with the more peripheral (i.e., nonhub) regions of the brain. Neurology ${ }^{\circledR}$ 2017;88:2107-2114

\section{GLOSSARY}

$\mathbf{C I}=$ cognitively impaired; $\mathbf{C P}=$ cognitively preserved; $\mathbf{D M N}=$ default-mode network; EDSS = Expanded Disease Severity Scale; $\mathbf{F P N}=$ frontoparietal network; $\mathbf{G M}=$ gray matter; $\mathbf{G M V}=$ gray matter volume; $\mathbf{H C}=$ healthy control; $\mathbf{M C I}=$ mildly cognitively impaired; $\mathbf{M S}=$ multiple sclerosis; $\mathbf{R O I}=$ region of interest; $\mathbf{R S N}=$ resting-state network; $\mathbf{T E}=$ echo time; $\mathbf{T R}=$ repetition time; $\mathbf{W M}=$ white matter.

Patients with multiple sclerosis (MS) commonly experience cognitive decline, which is most likely driven by functional network changes. ${ }^{1-3}$ Across neurologic disorders, changes in connectivity of especially the default-mode network (DMN) and frontoparietal network (FPN) have been linked to cognitive deficits. ${ }^{4-6}$ This preferential relationship might be explained by the fact that these networks contain the majority of highly connected regions, commonly described as functional hubs. ${ }^{7,8}$ In fact, such hub regions are essential for optimal cognitive function because they ensure efficient integration of information between different brain regions. ${ }^{9}$

Previous studies have reported both increased ${ }^{1,10,11}$ and decreased ${ }^{3,12,13}$ global connectivity levels of the DMN and FPN in relation to cognitive dysfunction in MS. This creates confusion about how these functional connectivity changes, either increased or decreased, may actually influence cognition. ${ }^{14}$ Apart from the directionality of these changes, it remains unclear whether these effects are due specifically to changes in within-network connectivity, connectivity with the rest of the brain, or changes in between-network connectivity.

From the Departments of Anatomy and Neurosciences (K.A.M., A.J.C.E., L.D., J.J.G.G., M.M.S.), Neurology (B.M.J.U.), and Radiology and Nuclear Medicine (F.B.), VUmc MS Center Amsterdam, Amsterdam Neuroscience Campus, VU University Medical Center, the Netherlands; and Institutes of Neurology and Healthcare Engineering (F.B.), University College London, London, UK.

Go to Neurology.org for full disclosures. Funding information and disclosures deemed relevant by the authors, if any, are provided at the end of the article. 
Although a few studies have separately investigated such connectivity changes between resting-state networks (RSNs) in MS, ${ }^{13,15}$ no study has investigated these 3 connectivity measures together in relation to cognitive dysfunction.

The aim of the present study was therefore to investigate changes in the DMN and FPN in patients with MS with different severities of cognitive impairment by specifically investigating within- and between-network connectivity as well as connectivity with the rest of the brain.

METHODS Participants. All participants were part of the Amsterdam MS cohort, ${ }^{2,16}$ which consists of 332 patients with clinically definite $\mathrm{MS}^{17}$ (age $48.1 \pm 11.0$ years, disease duration 14.6 years, range $4.6-45.9$ years) and 96 matched healthy controls (HCs; age $45.9 \pm 10.5$ years). Of the patients with MS, 243 patients were diagnosed with relapsing remitting MS, 53 patients with secondary progressive MS, and 36 with primary progressive MS.

Standard protocol approvals, registrations, and patient consents. The study protocol was approved by the local ethics review board, and all participants gave written informed consent before participation.

Neuropsychological assessment and cognitive groups. On the day of scanning, all participants underwent a neuropsychological assessment as previously described, ${ }^{2}$ consisting of an expanded Brief Repeatable Battery of Neuropsychological tests (see e-Methods at Neurology.org). Cognitive scores were regression-adjusted for normal effects of age, sex, and educational level on the basis of the effects observed in our HCs. ${ }^{18}$ These corrected cognitive test scores were used to compute $z$ scores relative to the mean and SD of HCs. Patients were defined cognitively impaired (CI) if they scored at least 2 SDs (i.e., $z$ score $<-2.0$ ) below HCs on at least 2 cognitive tests. Patients who did not fulfill the criteria for CI but who scored at least 1.5 SDs below $\mathrm{HCs}$ on at least 2 domains were defined mildly $\mathrm{CI}$ (MCI). Noteworthy, this term should not be confused with the clinical diagnosis of MCI caused by Alzheimer disease or other neurologic conditions. The remaining patients were defined cognitively preserved (CP).

MRI studies. MRI was performed on a 3T MRI system using an 8-channel head-coil (GE Signa-HDxt, Milwaukee, WI). All participants underwent a 3-dimensional T1-weighted inversion-prepared fast spoiled gradient recall sequence (repetition time [TR] 7.8 milliseconds, echo time [TE] 3 milliseconds, inversion time 450 milliseconds, flip angle $12^{\circ}$, sagittal 1.0 -mm sections, $0.94 \times 0.94-\mathrm{mm}^{2}$ in-plane resolution) for volumetric measurements and a 3-dimensional fluid-attenuated inversion-recovery sequence (FLAIR; TR 8,000 milliseconds, TE 125 milliseconds, inversion time 2,350 milliseconds, sagittal $1.2-\mathrm{mm}$ slices, $0.98 \times 0.98-\mathrm{mm}^{2}$ in-plane resolution) for lesion detection. Additionally, resting-state fMRI scans were acquired with whole-brain coverage and 202 volumes, of which the first 2 were discarded (echo planar imaging; TR $=200$ milliseconds, $\mathrm{TE}=35$ milliseconds, flip angle $20^{\circ}, 3 \mathrm{~mm}$ contiguous axial slices, $3.3 \times 3.3-\mathrm{mm}$ in-plane resolution).
Structural brain measures. White matter (WM) lesions were segmented on FLAIR images with the use of a previously described automated segmentation technique ${ }^{19}$ and used to calculate lesion volumes. The quality of the segmentation was manually assessed. Lesion filling (LEAP; LEsion Automated Preprocessing) was applied on the 3-dimensional T1 to minimize the effect of WM lesions on volumetric measurements. ${ }^{20}$ Total gray matter (GM) volume (GMV), total WM volume (WMV), and whole brain volume were then quantified with the lesion-filled T1-weighted image with SIENAX (part of FSL5, fsl.fmrib.ox.ac.uk). In addition, deep GMV (using FIRST, also part of FSL) and cortical GMV (by removing FIRST regions from the GM mask) were computed. All volumes were normalized for head size.

fMRI preprocessing. Preprocessing consisted of removal of nonbrain tissue, motion correction, spatial smoothing with a 5$\mathrm{mm}$ full width at half-maximum gaussian kernel, and high-pass temporal filtering equivalent to $0.01 \mathrm{~Hz}$. All resting-state fMRI scans were checked for registration errors and artifacts. The average motion did not exceed $3 \mathrm{~mm}$ (i.e., one voxel) for any participant; the average was $0.08 \mathrm{~mm}(0.05 \mathrm{~mm})$ with no difference between HCs and MS $(p=0.34)$. Registration parameters were calculated with the use of boundary-based registration between the fMRI and 3-dimensional T1 sequences and nonlinear registration between 3-dimensional T1 and standard space for subsequent atlas-based steps.

Regions of interest. The brain was separated into regions of interest (ROIs) with a custom-made native-space atlas as reported previously. ${ }^{2}$ The cortical atlas was derived from the standard-space Automated Anatomical Labeling atlas, which was coregistered to the participant's T1-weighted scan with inverted nonlinear registration parameters and nearest-neighbor interpolation. After registration, this cortical atlas was masked by a GM mask derived from SIENAX to ensure that only cortical GM was included. Deep GM structures (i.e., thalamus, caudate, putamen, pallidum, hippocampus, amygdala, and nucleus accumbens) were segmented with FIRST on the participant's T1-weighted scans and were added to the cortical atlas. The complete atlas was subsequently coregistered to the participant's resting-state fMRI scan with an inverted boundary-based registration matrix and nearest-neighbor interpolation to optimize registration. However, approaches such as bias-field measurements could further improve registration. After registration to fMRI, only those ROIs with at least 30\% voxels remaining after registration and masking with a custom-made fMRI mask were included in the analyses. This custom-made mask was created to remove any residual nonbrain tissue and to reduce the effect of echo planar imaging distortions by excluding voxels with signal intensities in the lowest quartile of the robust range. On the basis of these criteria, 12 ROIs were excluded, made up of orbitofrontal areas and nucleus accumbens. The final atlas therefore segmented the fMRI sequence into 80 regions for which mean time series were obtained.

To determine which atlas regions make up the DMN and FPN, the resting-state data were transferred to standard space to be able to run an independent component analysis with MELODIC. ${ }^{21}$ The concatenated fMRI dataset was decomposed into 42 components, with a clearly identifiable DMN and FPN. The following regions were selected for the DMN: medial superior frontal gyrus, posterior cingulate, angular gyrus, and precuneus. For the FPN, middle frontal gyrus, superior frontal gyrus, inferior parietal gyrus, angular gyrus, and precuneus were selected. Regions that were not part of the 
DMN or FPN were considered to be the rest of the brain, consisting mainly of nonhub regions.

Connectivity scores. For each participant, Pearson correlation coefficients were calculated between all 80 regions to construct connectivity matrices. Given the controversial nature of negative correlations, 2 approaches were used: either set to zero ${ }^{22,23}$ or maintained in the matrices. Each connectivity score was normalized on the basis of the mean and SD of each participant's connectivity matrix. In effect, these relative connectivity scores reflect whether connections were stronger (positive $z$ score) or weaker (negative $z$ score) than the average strength of all connections ( $z$ score $=0$ ) within an individual functional network. This measure was chosen to specifically look at the ranking of each brain region within its individual brain network, in doing so correcting for gross interparticipant variability in mean connectivity. Three different measures were calculated for the DMN and FPN: the connectivity within these RSNs, the connectivity of these RSNs with the rest of the brain, and the connectivity between these RSNs (figure 1). Subsequently, a post hoc regional analysis was performed for each significant network measure to identify which brain region within the DMN or FPN was most responsible statistically for the measured connectivity changes.

Statistical analyses. All statistical analyses in this study were performed with SPSS 21.0 (Chicago, IL). Variables were checked for normality with Kolmogorov-Smirnov testing and histogram inspection. Data were investigated with $\chi^{2}$ tests for categorical variables, Kruskal-Wallis tests for nonnormally distributed variables (Mann-Whitney $U$ tests as post hoc test), or general linear models for normally distributed variables. The Levene test for equality in variances was used to assess homoscedasticity. If this assumption was violated, the Welch test was used. To investigate whether the observed connectivity changes were specific for cognitive function, the analysis was repeated with the use of groups based on Expanded Disease Severity Scale (EDSS) scores $<3,3$ to 4 , and $>4$ (i.e., based on tertiles). All general linear models were corrected for age, sex, and education. To limit the number of statistical tests, group comparisons were limited to cognitive MS groups vs controls and CI vs CP. fMRI measures that were different between groups were correlated with structural measures and clinical data with the use of Pearson correlations for normally distributed variables or Spearman correlations otherwise. Only Bonferroni-corrected $p$ values were reported, and values of $p<0.05$ were considered significant.

RESULTS Demographical, clinical, and MRI data. Of all patients, 180 (54\%) were defined as CP, 65 as MCI (20\%), and 87 as CI (26\%) (table 1). As expected, CI patients were older relative to $\mathrm{HCs}$ and CP patients and had a longer symptom duration, higher EDSS, and lower level of education relative to CP patients. Additionally, MCI and CI patients had a lower level of education compared to HCs. There were more women in the CP group compared to HCs and CI patients. Among the volumetric measures, GMV and lesion volume differed between $\mathrm{CI}$ and CP patients, whereas WMV did not. All brain volumes were reduced in all MS groups compared to controls.

Cognitive profile of patients with MS. As expected, all cognitive domains were affected in CI patients compared to controls, with the strongest effects in information processing speed $(z$ score $=-2.54)$ and executive functioning $(z$ score $=-2.53$ ), followed by working memory $(z$ score $=-2.45$; table 2 ). Relative to HCs, CP patients had a worse performance only on information processing speed and working memory, while MCI and CI patients scored lower on all cognitive domains compared to controls, which was also seen for CI compared to CP patients. Connectivity of the DMN and FPN. For the DMN, connectivity with the rest of the brain was increased only in CI patients compared to HCs $\left(p_{\text {corr }}=0.04\right)$

Figure $1 \quad$ DMN and FPN measures

\section{A. Connectivity within RSNs}
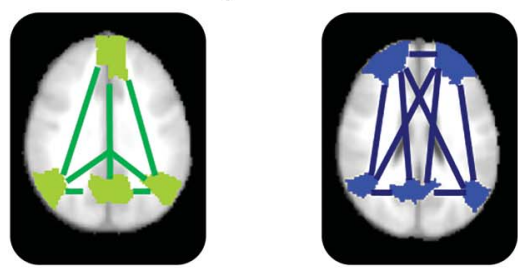

\section{B. Connectivity between RSNs}
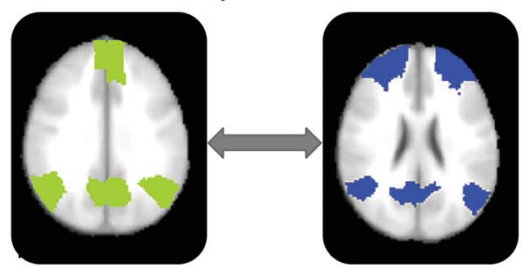

C. Connectivity between RSNs and rest of the brain
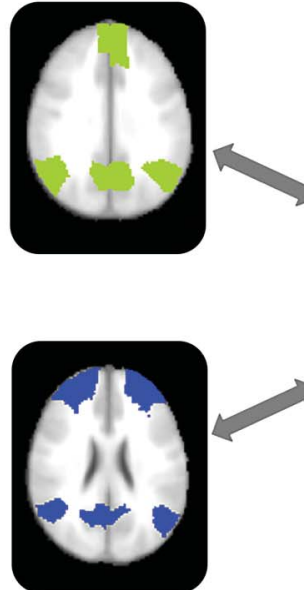

Within-DMN (green) and within-FPN (blue) connectivity (A) and connectivity between these networks (B). Connectivity between both the DMN and FPN with the rest of the brain (e.g., all other regions of the Automated Anatomical Labeling atlas except for the DMN and FPN regions; C). DMN = default-mode network; FPN = frontoparietal network; RSN = resting-state network. 
Table 1 Demographic, clinical, and MRI data of included participants

\begin{tabular}{|c|c|c|c|c|c|}
\hline & HCs & $\mathrm{CP}$ & $\mathrm{MCl}$ & $\mathrm{Cl}$ & Overall $p$ value \\
\hline Patients, $n$ & 96 & 180 & 65 & 87 & \\
\hline RRMS/SPMS/PPMS, n & & $148 / 21 / 11$ & $46 / 6 / 13$ & $49 / 26 / 12^{a}$ & $<0.01^{\mathrm{b}}$ \\
\hline Age, $y^{c}$ & 6 (10.5) & 46 (10.5) & 49 (12.2) & $51(10.7)^{a, d}$ & $<0.01^{\mathrm{e}}$ \\
\hline $\mathrm{F} / \mathrm{M}, \mathrm{n}$ & $56 / 40$ & $132 / 48^{d}$ & $42 / 23$ & $52 / 35^{a}$ & $0.04^{b}$ \\
\hline Level of education, $y^{f}$ & $6(1-7)$ & $6(1-7)$ & $4(1-7)^{d}$ & $4(2-7)^{a, d}$ & $<0.01^{9}$ \\
\hline Symptom duration, $y^{f}$ & & $10(5-34)$ & $13(5-35)$ & $18(5-46)^{a}$ & $0.01^{\mathrm{g}}$ \\
\hline EDSS score ${ }^{f}$ & & $3(0-8)$ & $3(0-8)$ & $4(2-8)^{a}$ & $<0.01^{\mathrm{g}}$ \\
\hline NBV, Lc & $1.52(0.07)$ & $1.48(0.06)^{d}$ & $1.46(0.07)^{d}$ & $1.40(0.09)^{a, d}$ & $<0.01^{h}$ \\
\hline NGMV, Lc & $0.82(0.05)$ & $0.80(0.05)^{d}$ & $0.79(0.05)^{d}$ & $0.75(0.06)^{\mathrm{a}, \mathrm{d}}$ & $<0.01^{h}$ \\
\hline NDGMV, $\mathrm{mL}^{\mathrm{c}}$ & $62.9(3.7)$ & $58.6(5.2)^{d}$ & $56.2(5.8)^{d}$ & $50.9(7.8)^{\mathrm{a}, \mathrm{d}}$ & $<0.01^{h}$ \\
\hline NWMV, Lc & $0.70(0.03)$ & $0.68(0.03)^{d}$ & $0.67(0.03)^{d}$ & $0.65(0.04)^{d}$ & $<0.01^{\mathrm{e}}$ \\
\hline $\begin{array}{l}\text { Normalized total lesion } \\
\text { load, } \mathrm{mL}^{f}\end{array}$ & & $10.8(0.8-83.5)$ & $14.3(1.8-56.9)$ & $24.0(2.1-94.7)^{a}$ & $<0.01^{9}$ \\
\hline
\end{tabular}

Abbreviations: $\mathrm{Cl}=$ cognitively impaired; $\mathrm{CP}=$ cognitively preserved; EDSS $=$ Expanded Disease Severity Scale; $\mathrm{HC}=$ healthy control; $\mathrm{MCl}=$ mild cognitively impaired; $\mathrm{NBV}=$ normalized brain volume; NDGMV = normalized deep gray matter volume; NGMV = normalized gray matter volume; NWMV = normalized white matter volume; PPMS = primary progressive multiple sclerosis; RRMS = relapsing remitting multiple sclerosis; SPMS = secondary progressive multiple sclerosis.

For the statistical tests conducted to obtain $p$ values, all assumptions were met.

${ }^{a}$ Difference between $\mathrm{Cl}$ and $\mathrm{CP}$.

b The $\chi^{2}$ test.

${ }^{\mathrm{c}}$ Results are expressed as mean (SD) for normally distributed variables.

${ }^{\mathrm{d}}$ Difference with HCs.

${ }^{\mathrm{e}}$ Analysis of variance.

${ }^{f}$ Results are expressed as median (range).

${ }^{9}$ Kruskal-Wallis test.

${ }^{\mathrm{h}}$ Welch test.

and CP patients $\left(p_{\text {corr }}=0.004\right.$; figure 1$)$, while within-DMN connectivity was not different between groups. For the FPN, similar results were observed; i.e., increased connectivity with the rest of the brain was seen only in CI patients compared to HCs $\left(p_{c o r r}=0.004\right)$ and CP patients $\left(p_{\text {corr }}=0.004\right.$; figure 2), while within-FPN connectivity did not differ between groups (figure 3). Connectivity between the DMN and FPN was not different between any of the groups. Results did not change when negative correlations were included in the analysis or when normalized brain volume was added as a covariate. Additionally, group differences in brain volumes were not mediated by functional

Table 2 Cognitive profile of $\mathrm{CP}$ and $\mathrm{Cl}$ patients

$\begin{array}{llllll} & \text { HCs } & \text { CP } & \text { MCI } & \text { CI } & \text { Overall } p_{\text {corr value }} \\ \text { Executive functioning } & 0.00(0.73) & -0.20(0.75) & -1.10(1.02)^{\mathrm{a}} & -2.72(2.21)^{\mathrm{a}, \mathrm{b}} & <0.001^{\mathrm{c}} \\ \text { Verbal memory } & 0.00(0.90) & 0.02(0.88) & -0.56(1.01)^{\mathrm{a}} & -1.48(1.05)^{\mathrm{a}, \mathrm{b}} & <0.001^{\mathrm{d}} \\ \text { Information processing speed } & 0.00(1.00) & -0.36(0.94)^{\mathrm{a}} & -1.59(0.68)^{\mathrm{a}} & -2.57(1.17)^{\mathrm{a}, \mathrm{b}} & <0.001^{\mathrm{c}} \\ \text { Verbal fluency } & 0.00(1.00) & -0.05(0.95) & -0.74(0.80)^{\mathrm{a}} & -1.16(0.96)^{\mathrm{a}, \mathrm{b}} & <0.001^{\mathrm{d}} \\ \text { Visuospatial memory } & 0.00(0.94) & -0.17(1.01) & -0.82(1.05)^{\mathrm{a}} & -1.40(1.11)^{\mathrm{a}, \mathrm{b}} & <0.001^{\mathrm{d}} \\ \text { Working memory } & 0.00(0.85) & -0.39(0.79)^{\mathrm{a}} & -1.22(1.01)^{\mathrm{a}} & -2.45(1.94)^{\mathrm{a}, \mathrm{b}} & <0.001^{\mathrm{c}} \\ \text { Attention } & 0.00(0.66) & -0.18(0.65) & -0.98(1.05)^{\mathrm{a}} & -1.45(1.50)^{\mathrm{a}, \mathrm{b}} & <0.001^{\mathrm{c}}\end{array}$

Abbreviations: $\mathrm{Cl}$ = cognitively impaired; $\mathrm{CP}=$ cognitively preserved; $\mathrm{HC}=$ healthy control; $\mathrm{MCl}=$ mildly cognitively impaired.

Mean z scores were obtained with cognitive scores of HCs used as reference. A general linear model or Welch test was used to assess group differences in cognitive performance. Bonferroni-corrected $p$ values are reported.

a Difference with HCs.

${ }^{b}$ Difference between $\mathrm{Cl}$ and $\mathrm{CP}$.

${ }^{c}$ Welch test.

d Linear model. 
Figure 2 Connectivity between the DMN and the rest of the brain

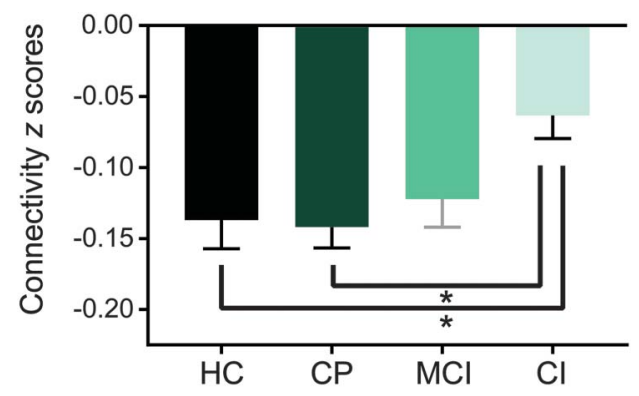

$\mathrm{Cl}$ patients showed increased connectivity between the $\mathrm{DMN}$ and the rest of the brain compared to $\mathrm{CP}$ patients and $\mathrm{HCs}$, reflected by higher $\mathrm{z}$ scores (i.e., less negative). Differences are indicated $\left(*{ }^{*} p_{\text {corr }}<0.05\right)$, and error bars reflect SEM. $\mathrm{Cl}=$ cognitively impaired; $\mathrm{CP}=$ cognitively preserved; $\mathrm{DMN}=$ default-mode network; $\mathrm{HC}=$ healthy control; $\mathrm{MCl}=$ mildly cognitively impaired.

connectivity. Although group effects were observed for the EDSS groups, post hoc comparisons did not survive multiple-comparison correction.

Post hoc regional connectivity changes. Post hoc analysis revealed increased connectivity with the rest of the brain for the inferior parietal $\left(p_{\text {corr }}=0.01\right)$, posterior cingulate $\left(p_{c o r r}=0.03\right)$, and angular gyri $\left(p_{c o r r}=0.01\right)$. These changes were observed only in CI patients, in whom increased connectivity was observed for the posterior cingulate and angular gyrus with the rest of the brain relative to $\mathrm{CP}$ $\left(p_{\text {corr }}=0.04\right.$ and $p_{\text {corr }}=0.004$, respectively $)$ and for the inferior parietal gyrus relative to $\mathrm{HCs}$ $\left(p_{\text {corr }}<0.008\right)$.

\section{Figure 3 Connectivity between the FPN and the rest of the brain}

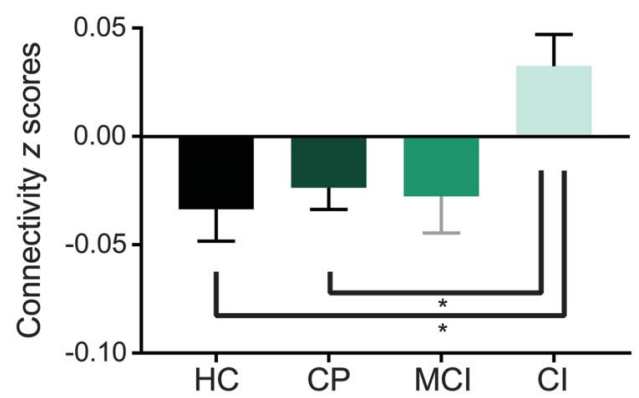

In $\mathrm{Cl}$ patients, increased connectivity, reflected by higher $z$ scores, was observed between the FPN and the rest of the brain compared to $\mathrm{CP}$ patients and HCs. This indicates that connections between the FPN and all other regions became stronger than the average strength of all connections together of the functional network. Differences are indicated $\left(* p_{\text {corr }}<0.05\right)$, and error bars reflect SEM. $\mathrm{Cl}=$ cognitively impaired; $\mathrm{CP}=$ cognitively preserved; FPN = frontoparietal network; $\mathrm{HC}=$ healthy control; $\mathrm{MCl}=$ mildly cognitively impaired.
Correlates of DMN and FPN connectivity. In MS, increased connectivity scores with the rest of the brain correlated only with worse cognition, including information processing speed and working memory for both the DMN-brain $(r=-0.179$ and $r=$ -0.171 , respectively) and FPN-brain $(r=-0.176$ and $r=-0.175$, respectively) and attention $(r=$ -0.152) only for the FPN-brain. Lower normalized deep GMV was associated with higher connectivity with the rest of the brain of both the DMN $(r=$ $-0.184)$ and FPN $(r=-0.149)$. Higher EDSS scores were associated with higher connectivity between the DMN and the rest of the brain $(r=$ 0.142) (all $p_{\text {corr }}<0.05$; table e-1).

DISCUSSION In this study, we examined the cognitive relevance of the hub-rich and cognitively important DMN and FPN in a large cohort of patients with MS separated into groups of different severities of cognitive impairment. Of the 332 patients, $46 \%$ were defined as either MCI or CI, involving multiple cognitive domains, with executive function and information processing speed being most severely affected. Despite such a high incidence in MS and a large body of literature investigating cognitive dysfunction, the specific network properties that drive cognitive deterioration in MS remain unclear. We specifically investigated hub network functioning by separately assessing connectivity within each network, of each network with the rest of the brain, and between the 2 networks. Our results showed that these 2 networks remained internally unchanged in terms of within- and between-network connectivity but showed increased connectivity with the rest of the brain in patients with more severe cognitive impairment.

Regionally, we observed that connectivity increases of the DMN and FPN with the rest of the brain were driven mostly by the inferior parietal, posterior cingulate, and angular cortices. Previous studies have also highlighted the cognitive relevance of structural ${ }^{16,24}$ and functional ${ }^{1,25}$ changes of especially the posterior cingulate gyrus, the most strongly connected hub in the brain. Properties of the human brain network facilitate efficient communication between separate brain regions by strong local connectivity, enabling local processing, and efficient long-distance connections, enabling integration of information. ${ }^{26}$ Because hubs are thought to guide most of this information flow in brain networks, they are likely to relate to cognitive function. ${ }^{9}$ In light of this perspective, our findings might indicate that especially the functional integration between hub regions and more provincial regions (e.g., non-DMN and non-FPN regions) has been altered 
in CI patients with MS. GM and WM damage throughout the brain may result in a lowered potential for local information processing, forcing the processing load away from peripheral areas toward more centrally located hubs, potentially inducing an inefficient network balance. ${ }^{27}$ The functional and structural properties of these hub regions might therefore predispose them for pathology, more so than nonhub regions. ${ }^{28}$ Interestingly, hubs seem to be especially sensitive to such a network pathology across many different neurologic disorders, stressing the clinical relevance of normal hub functioning. ${ }^{28-30}$ To ensure comparison with other studies, we defined the hub-rich DMN and FPN on the basis of an anatomic atlas. However, future studies are needed to select hub and nonhub regions in a fully data driven way.

Our finding is supported by previous studies in which increased connectivity was most often associated with worse cognitive performance in MS, possibly indicating a maladaptive process. ${ }^{1,2,25}$ However, these findings seem to be in contrast to the functional reorganization hypothesis in which it would be expected that patients with more severe structural damage would show decreased functional connectivity. ${ }^{14}$ The histopathologic correlate of the observed increased connectivity remains elusive; however, this could include an abnormally low inhibitory, GABA-based activity of interneurons. In MS, there seems to be a reduction in the population of interneurons, ${ }^{31,32}$ although it is unclear whether specifically interneurons are more sensitive to MS pathology. Nevertheless, a decrease in GABAergic neurotransmission has been observed in MS. ${ }^{33}$ In fact, previous neural mass model-based studies have shown that even a small loss of interneurons, and thus a small drop in inhibitory activity, can induce a very large functional effect on hubs and the entire brain network. ${ }^{34}$ However, given that excitatory neurons are most likely also affected by MS, it seems that a lower inhibitory activity could not be the only mechanism that underlies functional brain changes. For instance, a late and possibly ineffective attempt at beneficial functional reorganization cannot be excluded as an explanation for our results at this time. ${ }^{14}$ Such an attempt at plasticity could present itself by an outreach of hub-rich networks such as the DMN and FPN toward nonhub areas to facilitate information processing and transfer between hub and nonhub regions. Unfortunately, the cross-sectional design of almost all studies in the literature limits strong causal inferences, highlighting the need for longitudinal data.

In contrast to previous studies, we found no changes in within-network connectivity. ${ }^{10,13,35,36}$ Those studies, however, have been limited mostly to more conventional methodologies and usually do not separate CI and CP patient groups. Previous studies on pediatric and adult MS showed a cognitively relevant increased connectivity of the DMN with other brain networks, but not with the FPN specifically. ${ }^{15,13}$ Together, these studies seem to support our findings of increased RSN connectivity with other, nonhub, brain networks and no between-network changes.

Exploring the underlying mechanisms of cognitive deficits could help to define whether patients are genuinely CP or CI. Our data support a more conservative criterion of $-2 \mathrm{SD}$ on at least 2 tests because no network changes were observed in the MCI group. The normal functioning of both the DMN and FPN is known to be important for a broad range of cognitive demands. ${ }^{4}$ The increased connectivity of FPN with the rest of the brain was associated mostly with attention deficits in our sample, and the FPN has been implicated as a source of attention control before. ${ }^{23,37}$ Interestingly, given the involvement of frontal regions, the lack of a relation with our executive function domain is striking. This might be explained by the fact that the concept-shifting task, which makes up the executive functioning domain in our study, also has a prominent information processing speed component. The DMN is usually considered to be engaged during unconstrained cognitive processes, but its relevance for working memory has been shown before. ${ }^{38}$ Furthermore, functional connectivity changes of the DMN and FPN correlated with normalized deep GMV, the main structural correlate of cognitive dysfunction in MS. ${ }^{2}$ In addition, the main descriptive predictor of cognitive dysfunction, a lower level of education, was confirmed in the present study, which supports the notion of a cognitive reserve in MS.

We showed that functional connectivity levels of the DMN and FPN can distinguish cognitive phenotypes in MS. In CI patients with MS, these hub-rich networks show an increased level of connectivity, but only with peripheral, nonhub regions of the brain. This seemingly negative change in network balance needs to be investigated further in future longitudinal studies.

\section{AUTHOR CONTRIBUTIONS}

Drafting/revising the manuscript: all authors. Study concept or design: K.A.M., J.J.G.G., M.M.S. Acquisition of data: M.M.S. Analysis or interpretation of the data: K.A.M., A.J.C.E., M.M.S. Statistical analysis: K.A.M., A.J.C.E., M.M.S. Study supervision and coordination: J.J.G.G., M.M.S. Obtaining funding: B.M.J.U., F.B., J.J.G.G., M.M.S.

\section{STUDY FUNDING}

This study was supported by Dutch MS Research Foundation grants 08$650,13-820$, and 14-358e. 


\section{DISCLOSURE}

K. Meijer receives research support from a research grant from Biogen Idec. A. Eijlers receives research support from the Dutch MS Research Foundation, grant 14-358e. L. Douw receives research support from NWO Veni, grant 016.146.086, and Branco Weiss Fellowship from Society in Science. B. Uitdehaag has received consultation fees from Biogen Idec, Novartis, Merck-Serono, and Danone Research. F. Barkhof serves on the editorial boards of Brain, European Radiology, Journal of Neurology, Neurosurgery \& Psychiatry, Neurology, Multiple Sclerosis, and Neuroradiology, and serves as consultant for BayerShering Pharma, Sanofi-Aventis, Biogen-Idec, TEVA Pharmaceuticals, Genzyme, Merck-Serono, Novartis, Roche, Synthon, Jansen Research, and Lundbeck. J. Geurts serves on the editorial boards of Multiple Sclerosis Journal, BMC Neurology, Multiple Sclerosis International, and Neurology and the Scientific Advisory Board of the Dutch MS Research Foundation, MS Academia, and Merck-Serono and has served as a consultant for Merck-Serono, Biogen Idec, Novartis, Genzyme, and Teva Pharmaceuticals. M. Schoonheim receives research support from the Dutch MS Research Foundation, grant 13-820, and has received compensation for consulting services or speaker honoraria from ExceMed, Genzyme, Novartis, and Biogen. Go to Neurology.org for full disclosures.

Received July 28, 2016. Accepted in final form March 6, 2017.

\section{REFERENCES}

1. Hawellek DJ, Hipp JF, Lewis CM, Corbetta M, Engel AK. Increased functional connectivity indicates the severity of cognitive impairment in multiple sclerosis. Proc Natl Acad Sci USA 2011;108:19066-19071.

2. Schoonheim MM, Hulst HE, Brandt R, et al. Thalamus structure and function determine severity of cognitive impairment in multiple sclerosis. Neurology 2015;84: 776-783.

3. Louapre C, Perlbarg V, García-Lorenzo D, et al. Brain networks disconnection in early multiple sclerosis cognitive deficits: an anatomofunctional study. Hum Brain Mapp 2014;35:4706-4717.

4. Rosazza C, Minati L. Resting-state brain networks: literature review and clinical applications. Neurol Sci 2011;32: 773-785.

5. Buckner RL, Andrews-Hanna JR, Schacter DL. The brain's default network anatomy, function, and relevance to disease. Ann NY Acad Sci 2008;1124:1-38.

6. Sylvester CM, Corbetta M, Raichle ME, et al. Functional network dysfunction in anxiety and anxiety disorders. Trends Neurosci 2012;35:527-535.

7. Sporns O. Contributions and challenges for network models in cognitive neuroscience. Nat Neurosci 2014; $17: 652-660$.

8. Buckner RL, Sepulcre J, Talukdar T, et al. Cortical hubs revealed by intrinsic functional connectivity: mapping, assessment of stability, and relation to Alzheimer's disease. J Neurosci 2009;29:1860-1873.

9. van den Heuvel MP, Sporns O. Network hubs in the human brain. Trends Cogn Sci 2013;17:683-696.

10. Faivre A, Rico A, Zaaraoui W, et al. Assessing brain connectivity at rest is clinically relevant in early multiple sclerosis. Mult Scler 2012;18:1251-1258.

11. Roosendaal SD, Schoonheim MM, Hulst HE, et al. Resting state networks change in clinically isolated syndrome. Brain 2010;133:1612-1621.

12. Leavitt VM, Paxton J, Sumowski JF. Default network connectivity is linked to memory status in multiple sclerosis. J Int Neuropsychol Soc 2014;20: 937-944.
13. Rocca MA, Valsasina P, Martinelli V, et al. Large-scale neuronal network dysfunction in relapsing-remitting multiple sclerosis. Neurology 2012;79:1449-1457.

14. Schoonheim MM, Meijer KA, Geurts JJG. Network collapse and cognitive impairment in multiple sclerosis. Front Neurol 2015;6:82.

15. Rocca MA, Valsasina P, Absinta M, et al. Intranetwork and internetwork functional connectivity abnormalities in pediatric multiple sclerosis. Hum Brain Mapp 2014;35: 4180-4192.

16. Steenwijk MD, Geurts JJG, Daams M, et al. Cortical atrophy patterns in multiple sclerosis are non-random and clinically relevant. Brain 2016;139:115-126.

17. Polman $\mathrm{CH}$, Reingold SC, Edan G, et al. Diagnostic criteria for multiple sclerosis: 2010 revisions to the McDonald criteria. J Neurol 2012;259:S8.

18. Amato MP, Portaccio E, Goretti B, et al. The Rao's brief repeatable battery and Stroop test: normative values with age, education and gender corrections in an Italian population. Mult Scler J 2006;12:787-793.

19. Steenwijk MD, Pouwels PJW, Daams M, et al. Accurate white matter lesion segmentation by $\mathrm{k}$ nearest neighbor classification with tissue type priors (kNN-TTPs). Neuroimage Clin 2013;3:462-469.

20. Chard DT, Jackson JS, Miller DH, Wheeler-Kingshott CAM. Reducing the impact of white matter lesions on automated measures of brain gray and white matter volumes. J Magn Reson Imaging 2010;32:223-228.

21. Damoiseaux JS, Rombouts SA, Barkhof F, et al. Consistent resting-state networks. Proc Natl Acad Sci USA 2006; 103:13848-13853.

22. Chai XJ, Castañán AN, Öngür D, Whitfield-Gabrieli S. Anticorrelations in resting state networks without global signal regression. Neuroimage 2012;59:1420-1428.

23. Fox MD, Snyder AZ, Vincent JL, Corbetta M, Van Essen DC, Raichle ME. The human brain is intrinsically organized into dynamic, anticorrelated functional networks. Proc Natl Acad Sci USA 2005;102:9673-9678.

24. Dineen RA, Vilisaar J, Hlinka J, et al. Disconnection as a mechanism for cognitive dysfunction in multiple sclerosis. Brain 2009;132:239-249.

25. Hulst HE, Schoonheim MM, Van Geest Q, Uitdehaag BM, Barkhof F, Geurts JJ. Memory impairment in multiple sclerosis: relevance of hippocampal activation and hippocampal connectivity. Mult Scler 2015;21: 1705-1712.

26. Sporns O. Network attributes for segregation and integration in the human brain. Curr Opin Neurobiol 2013;23: 162-171.

27. Stam CJ. Modern network science of neurological disorders. Nat Rev Neurosci 2014;15:683-695.

28. Crossley NA, Mechelli A, Scott J, et al. The hubs of the human connectome are generally implicated in the anatomy of brain disorders. Brain 2014;137:23822395.

29. de Haan W, Pijnenburg YAL, Strijers RL, et al. Functional neural network analysis in frontotemporal dementia and Alzheimer's disease using EEG and graph theory. BMC Neurosci 2009;10:101-113.

30. Agosta F, Sala S, Valsasina P, et al. Brain network connectivity assessed using graph theory in frontotemporal dementia. Neurology 2013;81:134-143.

31. Nicholas R, Magliozzi R, Campbell G, Mahad D, Reynolds R. Temporal lobe cortical pathology and inhibitory 
GABA interneuron cell loss are associated with seizures in multiple sclerosis. Mult Scler J 2016;22:25-35.

32. Gilmore CP, Deluca GC, Bö L, et al. Spinal cord neurona pathology in multiple sclerosis. Brain Pathol 2009;19: 642-649.

33. Dutta R, McDonough J, Yin X, et al. Mitochondrial dysfunction as a cause of axonal degeneration in multiple sclerosis patients. Ann Neurol 2006;59:478-489.

34. Denève $S$, Machens CK. Efficient codes and balanced networks. Nat Neurosci 2016;19:375-382.

35. Zhou F, Zhuang Y, Gong $\mathrm{H}$, et al. Altered inter-subregion connectivity of the default mode network in relapsing remitting multiple sclerosis: a functional and structural connectivity study. PLoS One 2014;9:e101198.

36. Tewarie P, Schoonheim MM, Stam CJ, et al. Cognitive and clinical dysfunction, altered MEG resting-state networks and thalamic atrophy in multiple sclerosis. PLoS One 2013;8:e69318.

37. Dosenbach NU, Fair DA, Miezin FM, et al. Distinct brain networks for adaptive and stable task control in humans. Proc Natl Acad Sci USA 2007;104:11073-11078.

38. Hampson M, Driesen NR, Skudlarski P, Gore JC, Constable RT. Brain connectivity related to working memory performance. J Neurosci 2006;26:13338-13343.

\section{Registration Open for 2017 Sports Concussion Conference in Jacksonville, FL}

Stay up-to-date and discover the latest information on the prevention, diagnosis, and treatment of sports concussion through interactive hands-on workshops, debates, and other engaging formats led by top experts. Early registration discounts end June 15. Visit AAN.com/view/ConcussionConference today.

\section{We're Looking for the Next Generation of Leaders- Apply for Emerging Leaders Program!}

Are you looking to take your career to the next level? We're looking for you! Applications are now open for a prestigious and interactive six-month program designed to identify, engage, and mentor AAN members who are less than 10 years out of residency or training. Learn more and apply by the July 1 deadline at $A A N$.com/view/ELP.

\section{Save These Dates for AAN CME Opportunities!}

Mark these dates on your calendar for exciting continuing education conferences by the American Academy of Neurology. Learn more at AAN.com/conferences.

\section{Sports Concussion Conference}

- July 14-16, 2017, Jacksonville, FL, at the Hyatt Regency Jacksonville Riverfront

\section{AAN Fall Conference}

- October 20-22, 2017, Las Vegas, NV, at The Cosmopolitan of Las Vegas

Breakthroughs in Neurology Conference

- January 12-15, 2018, Orlando, FL, at the Caribe Royale Orlando 


\section{Neurology}

\section{Increased connectivity of hub networks and cognitive impairment in multiple sclerosis}

Kim A. Meijer, Anand J.C. Eijlers, Linda Douw, et al.

Neurology 2017;88;2107-2114 Published Online before print May 3, 2017

DOI 10.1212/WNL.0000000000003982

\section{This information is current as of May 3, 2017}

$\begin{array}{ll}\begin{array}{l}\text { Updated Information \& } \\ \text { Services }\end{array} & \begin{array}{l}\text { including high resolution figures, can be found at: } \\ \text { http://www.neurology.org/content/88/22/2107.full.html }\end{array} \\ \text { Supplementary Material } & \begin{array}{l}\text { Supplementary material can be found at: } \\ \text { http://www.neurology.org/content/suppl/2017/05/03/WNL.0000000000 } \\ 003982 . D C 1\end{array} \\ & \text { This article cites } 38 \text { articles, } 12 \text { of which you can access for free at: } \\ \text { http://www.neurology.org/content/88/22/2107.full.html\#\#ref-list-1 } & \text { This article, along with others on similar topics, appears in the } \\ \text { following collection(s): } \\ \text { fMRI } \\ \text { http://www.neurology.org//cgi/collection/fmri } \\ \text { Multiple sclerosis } \\ \text { http://www.neurology.org//cgi/collection/multiple_sclerosis } \\ \text { Information about reproducing this article in parts (figures,tables) or in } \\ \text { its entirety can be found online at: } \\ \text { http://www.neurology.org/misc/about.xhtml\#permissions } \\ \text { Permissions \& Licensing } & \text { Information about ordering reprints can be found online: } \\ & \text { http://www.neurology.org/misc/addir.xhtml\#reprintsus }\end{array}$

Neurology ${ }^{\circledR}$ is the official journal of the American Academy of Neurology. Published continuously since 1951, it is now a weekly with 48 issues per year. Copyright @ 2017 American Academy of Neurology. All rights reserved. Print ISSN: 0028-3878. Online ISSN: 1526-632X.

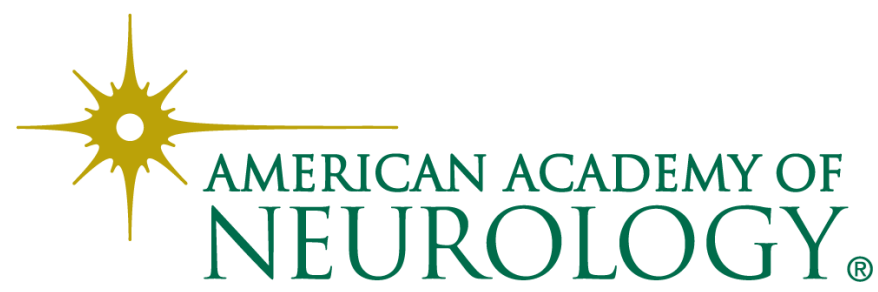

